\title{
Age-dependent auditory abnormalities
}

$\begin{array}{ll}\text { young } & \begin{array}{l}\text { Many people with 22q11.2 deletion } \\ \text { syndrome (22q11.2DS) develop } \\ \text { schizophrenia with psychotic }\end{array} \\ \text { mice showed } & \begin{array}{l}\text { symptoms (such as hallucinations) } \\ \text { in early adulthood. The relatively late } \\ \text { onset of these psychotic symptoms } \\ \text { compared with other features of }\end{array} \\ \text { increased } & \begin{array}{l}\text { 22q11.2DS is poorly understood. } \\ \text { thalamic Drd2 } \\ \text { expression and } \\ \text { TC signalling }\end{array} \\ \text { in a mouse model of 22q11.2DS } \\ \text { that disruptions in the activity of } \\ \text { thalamocortical (TC) projections to } \\ \text { the auditory cortex (AudCx) - sim- } \\ \text { ilar to those implicated in auditory } \\ \text { hallucinations - can be explained } \\ \text { by an age-dependent decline in the } \\ \text { expression of microRNA-338-3p } \\ \text { (miR-338-3p). }\end{array}$

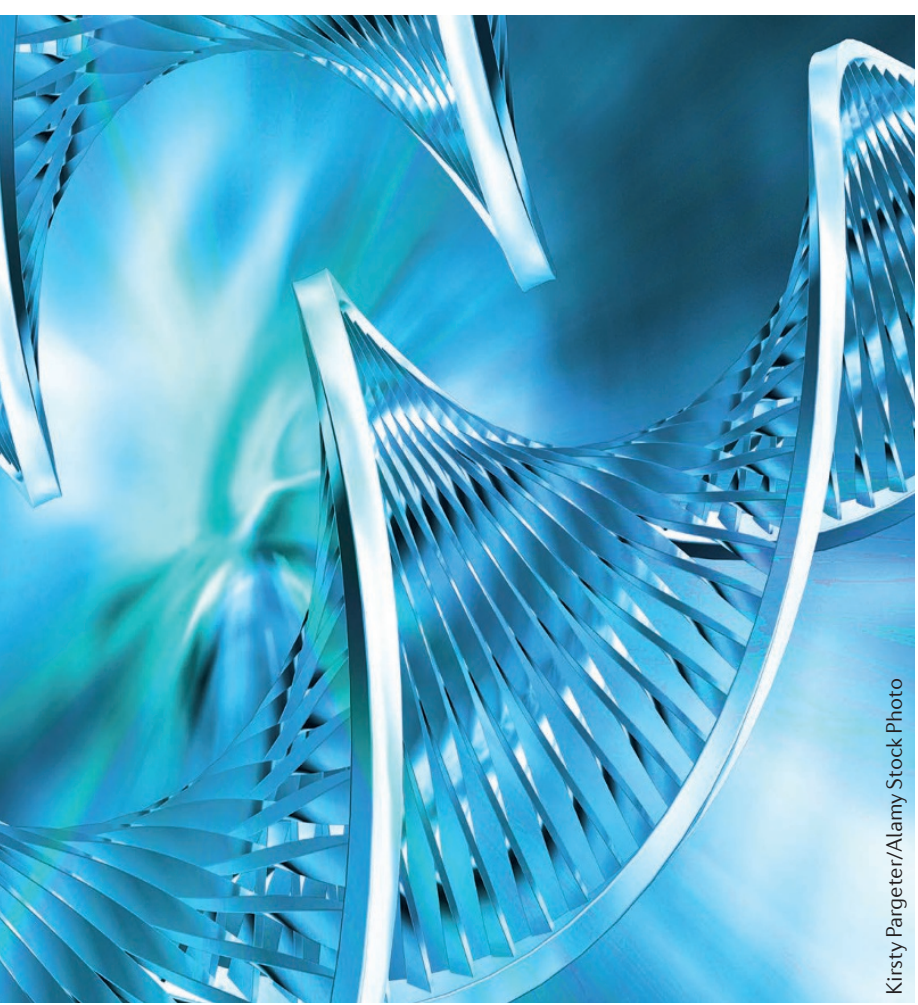

Previous research showed that haploinsufficiency of $\operatorname{Dgcr} 8$, which encodes a subunit of the microprocessor complex that processes microRNAs (miRNAs) and is one of the genes often deleted in 22q11.2DS, leads to an upregulation of dopamine D2 receptors (DRD2s) in thalamic neurons projecting to the AudCx and to deficits in TC signalling. In this study, Chun et al. took voltage-clamp recordings in acute slices from $D f(16) 1 /+$ mice (a model of 22q11.2DS), $D g c r 8^{+/-}$mice and wildtype controls, and assessed excitatory postsynaptic currents (EPSCs) in the AudCx that were evoked by thalamic stimulation. Stimulation-evoked EPSCs in mature - but not young - $D f(16) 1 /+$ or $D g c r 8^{+/-}$mice were smaller than those in controls but could be rescued by application of the antipsychotic and DRD2 antagonist haloperidol. In addition, mature (but not young) $D f(16) 1 /+$ and $D g c r 8^{+/-}$ mice showed increased thalamic expression of Drd2 mRNA. These data suggest that the deficits in TC signalling that are induced by a lack of DGCR8 are age-dependent.

To investigate how a loss of DGCR8 might increase DRD2 expression, the authors used microarray analysis and in vitro and in vivo experiments to identify miRNAs that are depleted in $D f(16) 1 /+$ or $\mathrm{Dgcr} 8^{+/-}$mice and that target the Drd2 transcript. Overexpression of one such miRNA, miR-338-3p, in excitatory thalamic neurons of mature $D f(16) 1 /+$ mice reduced thalamic Drd2 levels, rescued synaptic transmission deficits and reduced the sensitivity of AudCx EPSCs to haloperidol. Notably, miR-338-3p expression was found to decrease with age, reaching low levels in wild-type mice by maturity, and even lower levels in mature $\mathrm{Dgcr} 8^{+/-}$ mice. Therefore, an age-dependent decrease in miR-338-3p may mediate the TC deficits observed in mature Df(16) $1 /+$ mice.

Depletion of miR-338-3p levels in the thalamic medial geniculate nucleus of wild-type mice (through injection of a virus encoding a miR338-3p target sequence that acted as a 'sponge' for this miRNA) increased local Drd2 levels and led to haloperidol sensitivity - even in young mice. Moreover, young mir $338^{+/-}$mice showed increased thalamic Drd2 expression and TC signalling deficits that were rescued by haloperidol. Thus, a loss of miR-338-3p can induce the age-dependent TC deficits seen in $D f(16) 1 /+$ mice.

Together, these results suggest that deletion of $D g c r 8$ in 22q11.2DS leads to decreased processing of miR-338-3p, disinhibiting Drd2 processing and resulting in auditory TC deficits that might be associated with antipsychotic-sensitive auditory hallucinations. Interestingly, thalamic levels of miR-338-3p were shown to be lower in people with schizophrenia than in age-matched healthy controls, further implicating a loss of this miRNA in the disorder.

Natasha Bray

ORIGINAL ARTICLE Chun, S. et al. Thalamic miR338-3p mediates auditory thalamocortical disruption and its late onset in models of 22q11.2 microdeletion. Nat. Med. http://dx.doi. org/10.1038/nm.4240 (2016) FURTHER READING Javitt, D. C. \& Sweet, R. A. Auditory dysfunction in schizophrenia: integrating clinical and basic features. Nat. Rev. Neurosci. 16, 535-550 (2015) 